\title{
Do Small Headgroups of Phosphatidylethanolamine and Phosphatidic Acid Lead to a Similar Folding Pattern of the $\mathrm{K}^{+}$Channel?
}

\author{
Mobeen Raja
}

Received: 28 April 2011/Accepted: 27 June 2011/Published online: 10 July 2011

(C) The Author(s) 2011. This article is published with open access at Springerlink.com

\begin{abstract}
Phospholipid headgroups act as major determinants in proper folding of oligomeric membrane proteins. The $\mathrm{K}^{+}$-channel $\mathrm{KcsA}$ is the most popular model protein among these complexes. The presence of zwitterionic nonbilayer lipid phosphatidylethanolamine (PE) is crucial for efficient tetramerization and stabilization of KcsA in a lipid bilayer. In this study, the influence of PE on KcsA folding properties was analyzed by tryptophan fluorescence and acrylamide quenching experiments and compared with the effect of anionic phosphatidic acid (PA). The preliminary studies suggest that the small size and hydrogen bonding capability of the PE headgroup influences KcsA folding via a mechanism quite similar to that observed for anionic PA.
\end{abstract}

Keywords Assembly and stability - Hydrogen bonding KcsA potassium channel $\cdot$ Lipid bilayers $\cdot$ Phosphatidic acid · Phosphatidylethanolamine $\cdot$ Protein-lipid interaction

The zwitterionic phosphatidylethanolamine (PE) is the most abundant lipid of Escherichia coli membrane. It has a small headgroup (Fig. 1) that allows it to form nonbilayer structure. As a polar head group, PE creates a more viscous

\footnotetext{
M. Raja

Department of Biochemistry of Membranes, Centre for Biomembranes and Lipid Enzymology, Institute of Biomembranes, Utrecht University, Padualaan 8, $3584 \mathrm{CH}$ Utrecht, The Netherlands

\section{Raja $(\bowtie)$}

School of Molecular and Systems Medicine, Alberta Diabetes Institute, University of Alberta, 6126 HRIF East, Edmonton, AB T6G 2E1, Canada

e-mail: mraja@pmcol.ualberta.ca
}

lipid membrane compared to phosphatidylcholine (PC) (Dill and Stigter 1988). Because of its small size, the PE headgroup stimulates partial insertion of the catalytic domain of the enzyme leader peptidase into the membrane (van Klompenburg et al. 1998). PE also acts as a molecular chaperone in the folding of the integral membrane protein and it is therefore essential for Sec-dependent protein translocation (Rietveld et al. 1995).

To date, the potassium channel KcsA from Streptomyces lividans has been proven to be the most convenient protein to study the assembly and stability of oligomeric membrane proteins. Each monomer of KcsA consists of two transmembrane segments that are connected by the pore region, a membrane bound $\mathrm{N}$-terminus and a large $\mathrm{C}$-terminal domain hanging in the cytoplasm (Cortes et al. 2001). The conversion of monomeric subunits into an extremely stable tetramer makes KcsA a perfect model protein underlying protein-protein and protein-lipid dependent oligomerization and stabilization of membrane proteins. In particular, KcsA has been extensively studied for its association with different types of zwitterionic or anionic phospholipids in artificial membranes (Raja 2010c; Raja et al. 2007).

KcsA is expressed in relatively large amounts in Escherichia coli inner membrane (Heginbotham et al. 1998) that consists of $\sim 75 \%$ zwitterionic PE, $\sim 20 \%$ anionic PG and $\sim 5 \%$ cardiolipin (Raetz 1978). Both PE and PG provide the most efficient environment for KcsA channel assembly and tetrameric stability (van Dalen et al. 2002). The small headgroup of PE, which is quite similar to anionic lipid phosphatidic acid (PA) headgroup (Fig. 1), leads to a more favorable packing at the protein-lipid interface (Bogdanov et al. 2008; Demmers et al. 2003). PA is not an abundant lipid constituent of any living organism, but it is extremely important as an intermediate in the 


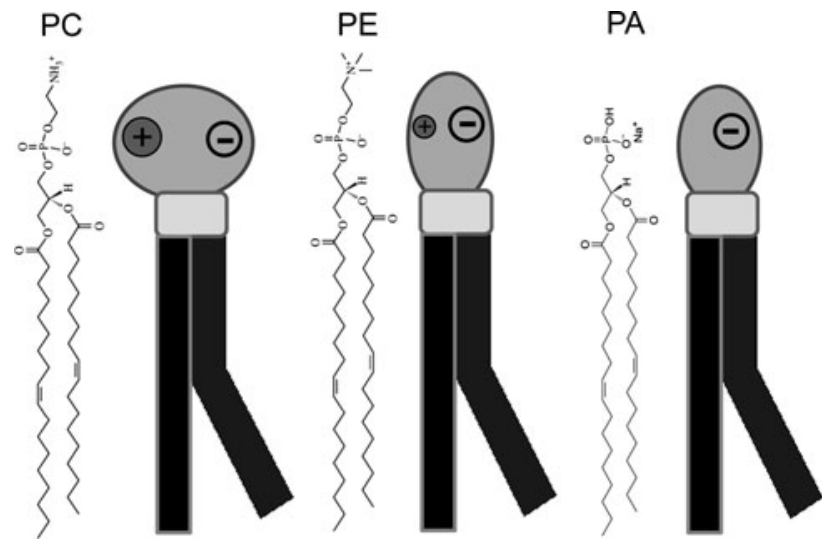

Fig. 1 The chemical structures and schematic diagram of the three membrane phospholipids depicting the charge distribution and headgroup size. The more or less cylindrical PC carries a zwitterionic headgroup on a glycerol with two fatty acyl chains (diacylglycerol), usually one unsaturated (bent). PE has a small headgroup and a conical shape and creates a stress in the bilayer. PA carries a negative charge and it also has a similar headgroup size as PE

biosynthesis of triacylglycerols and phospholipids and as a signaling molecule. It is the simplest diacylglycerophospholipid and the only one with a phosphomonoester as the headgroup (Boggs 1987). Previously, it has been shown that the presence of this lipid in the membrane stabilizes the tetrameric $\mathrm{KcsA} \mathrm{K}^{+}$channel to a great extent via deep insertion of lipid binding domains as a result of its small headgroup size and strong $\mathrm{H}$-bonding/electrostatic interaction with the protein (Raja et al. 2007). Because PA and $\mathrm{PE}$ share the same headgroup size or cone shape structure and H-bonding capabilities it is not yet clear whether both lipids affect the folding of integral membrane proteins in a similar fashion or not.

In the present study, KcsA tetramer folding patterns were compared in PE:PG (7:3 mol\%) and PC:PA (7:3 mol\%) lipid bilayers. Furthermore, the role of the $\mathrm{N}$-terminus in altering the tetramer conformation was also investigated in both lipid bilayers. The membrane mimicking cosolvent trifluoroethanol (TFE) was used as a powerful tool to examine the stabilizing and folding properties of KcsA tetramer as described in the earlier studies (Raja 2010c; Raja et al. 2007). The unfolding experiments suggest that small headgroup size as well as extensive hydrogen bonding properties of PE may result in a highly stable structure of KcsA tetramer, thus better resembling the unique folding pattern of KcsA-PA complex (Raja et al. 2007).

\section{Materials and Methods}

All chemicals were purchased from Sigma-Aldrich unless otherwise stated. 1,2-Dioleoyl-sn-glycero-3-phosphatidylethanolamine (DOPE), 1,2-dioleoyl-sn-glycero-3-phospho- glycerol (DOPG), 1,2-dioleoyl-sn-glycero-3-phosphocholine (DOPC), and 1,2-dioleoyl-sn-glycero-3-phosphatidic acid (DOPA) were purchased from Avanti Polar Lipids. For clarity, the names of these lipids are abbreviated to PE, PG, PC, and PA, respectively. 2,2,2-Trifluoroethanol (TFE) was obtained from Merck.

Protein Expression, Purification, and Reconstitution

WT- and $\Delta \mathrm{N}-\mathrm{KcsA}$ (lacking first 18 residues) proteins were expressed with an N-terminal His-tag from pT7-KcsA in Escherichia coli strain BL21 and purified according to the method described previously (Raja et al. 2007). The purity of proteins was assessed by sodium dodecyl sulfate-polyacrylamide gel electrophoresis (SDS-PAGE), and the protein concentration was determined by using standards of bovine serum albumin. Large unilamellar vesicles (LUVs) were prepared by extrusion (Hope et al. 1985) and proteins were reconstituted in PE:PG $(7: 3 \mathrm{~mol} \%)$ or PC:PA (7:3 mol\%) lipid mixtures with a 1:1000 protein:lipid molar ratio, and the resultant proteoliposomes were obtained as described previously (Raja et al. 2007).

\section{SDS-PAGE and Stability Assay}

To control the equal amounts of proteins in all experiments the amount of protein reconstituted into the proteoliposomes was determined by Coomasie Blue staining using standards of bovine serum albumin. Small aliquots of proteoliposomes were incubated with variable concentrations of TFE for $1 \mathrm{~h}$ at room temperature. Samples were mixed with an electrophoresis sample buffer $(50 \mathrm{mM}$ Tris- $\mathrm{HCl}$ [pH 6.8], 50\% glycerol, $0.01 \%$ bromophenol blue and $10 \%$ SDS) and run on $15 \%$ acrylamide gel in the presence of $0.1 \%$ SDS. Silver nitrate-stained gels were scanned by a densitometer (Bio-Rad) and quantified with the program Quantity One (Bio-Rad). For stability assay, the amount of tetramer (\%) was calculated according to the procedure described previously (Raja et al. 2007). Values are given as means \pm SD. Significance $(P<0.05)$ was calculated using Student's $t$-test unless otherwise mentioned.

\section{Tryptophan Fluorescence and Acrylamide Quenching}

Proteoliposome samples were treated with TFE at desired concentrations. Fluorescence experiments were performed using a QuantaMaster QM-1/2005 spectrofluorometer (Photon Technology, Princeton, NJ), fitted with a xenon flash lamp at room temperature and Trp fluorescence spectra were collected according to the procedure described previously (Raja et al. 2007). Acrylamide quenching experiments were performed to determine the accessibility of Trp residues in lipid bilayers either in the presence or absence of 
TFE. The quenching data was analyzed by a Stern-Volmer equation as described previously (Raja et al. 2007).

\section{Results}

First, the intrinsic stability of KcsA tetramer reconstituted in a PE:PG $(7: 3 \mathrm{~mol} \%)$ lipid bilayer was determined by conventional gel electrophoresis. Figure 2a represents SDS gels illustrating tetramer dissociation pattern in WT and
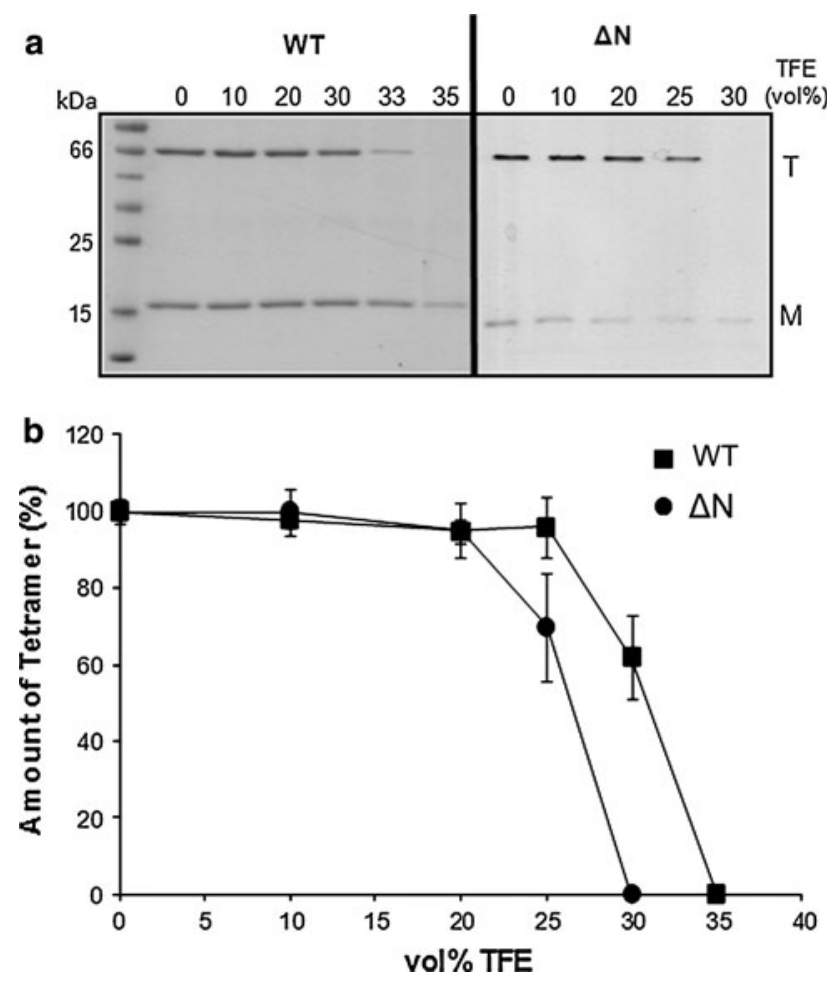

Fig. 2 TFE-induced tetramer dissociation of WT and $\Delta \mathrm{N}-\mathrm{Kcs} A$ in PE:PG (7:3 mol.\%) lipid bilayers. a TFE-treated samples were analyzed by SDS-PAGE. Tetrameric (T) and monomeric (M) KcsA are indicated; and a protein marker (in $\mathrm{kDa}$ ) is shown on the right. b Gels shown panel a were quantified for WT and $\Delta \mathrm{N}-\mathrm{Kcs}$. The intensities of tetramer bands were assigned as a relative value of $100 \%$ observed for a TFE-untreated (0 vol\% TFE) sample. Data points correspond to the average $\pm \mathrm{SD}$ of three experiments
$\Delta \mathrm{N}-\mathrm{KcsA}$ as a function of TFE (vol\%). Either in the absence or presence of TFE, the amount of WT-KcsA monomer is relatively higher than $\Delta \mathrm{N}-\mathrm{Kcs} \mathrm{A}$ monomer indicating low solubility of the monomeric $\Delta \mathrm{N}-\mathrm{KcsA}$ as discussed in the previous studies (Raja 2010a, b, c). For WT-KcsA tetramer, which typically runs at $\sim 68 \mathrm{kDa}$, $\sim 35 \mathrm{vol} \%$ TFE was required to dissociate the tetramer into its monomeric subunits $(\sim 18 \mathrm{kDa})$. However, $\Delta \mathrm{N}-\mathrm{Kcs} \mathrm{A}$ was completely destabilized at $\sim 30$ vol\% TFE indicating once again that the $\mathrm{N}$-terminus contributes to the stabilizing effect of full-length KcsA as shown in the previous studies (Raja 2010a, b, c). The disappearance of monomeric $\Delta \mathrm{N}-\mathrm{KcsA}$ is an indicative of protein aggregation at either low or high TFE concentrations that cannot be seen on the SDS gel (Raja et al. 2007). Figure 2b demonstrates the quantification of representative SDS gels shown in Fig. 2a in which the amount of tetrameric fraction (\%) at various TFE concentrations was calculated relative to the total amount of tetramer present in the absence of TFE ( 0 vol\%). These results indicate that the N-terminus significantly stabilizes the tetramer in a PE:PG lipid bilayer similar to PC:PG bilayers (Raja 2010b).

To investigate the folding properties of WT and $\Delta \mathrm{N}$ KcsA in a PE:PG lipid bilayer tryptophan (Trp) fluorescence experiments were performed. The strong sensitivity of Trp fluorescence has been proven to be a powerful tool in investigating KcsA folding properties in different lipid bilayer systems (Raja 2010b; Raja et al. 2007). For WTKcsA, Trp fluorescence emission spectra in the absence and presence of various concentrations of TFE (vol\%) are shown in Fig. 3a and the calculated values of emission maxima are complied in Table 1. It is interesting to observe that 5-10 vol\% TFE increases Trp fluorescence intensity, thus demonstrating the shielding effect on Trp residues from the local environment. Further increasing TFE concentration (20-30 vol\%) resulted in decrease in fluorescence or fluorescence quenching (25-33\%) followed by redshifts in emission maxima indicating an increase in hydrophilicity or increased accessibility of Trp residues to their local environment possibly due to protein unfolding (Raja 2010b; Raja et al. 2007). Using higher TFE
Fig. 3 Effect of TFE on Trp fluorescence of WT-KcsA (a) and $\Delta \mathrm{N}-\mathrm{K} \operatorname{csA}$ (b) in PE:PG (7:3 mol\%) bilayers. Representative emission spectra are shown. Samples were investigated in the absence or presence of varying concentrations of TFE (vol\%) as indicated
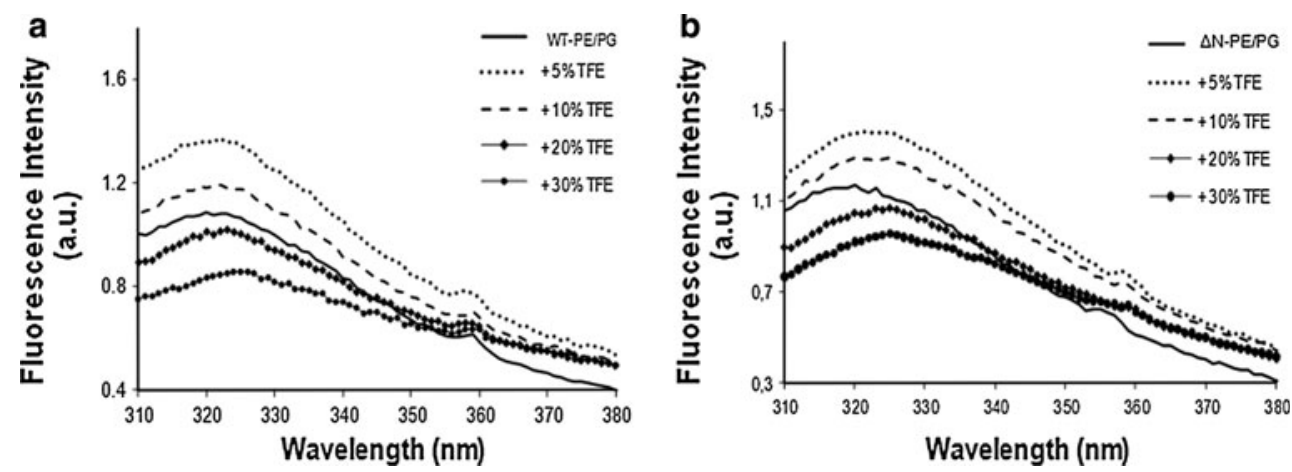
Table 1 Effects of TFE on folding properties of WT and $\Delta \mathrm{N}-\mathrm{KcsA}$ in a PE:PG (7:3 mol\%) lipid bilayer

\begin{tabular}{|c|c|c|c|c|c|c|c|c|c|c|}
\hline \multirow{2}{*}{$\frac{\text { Samples }}{\text { TFE (vol\%) }}$} & \multicolumn{5}{|c|}{ Wavelength (nm) ${ }^{\mathrm{a}}$} & \multicolumn{5}{|c|}{$K_{\mathrm{SV}}\left(\mathrm{M}^{-1}\right)^{\mathrm{b}}$} \\
\hline & 0 & 5 & 10 & 20 & 30 & 0 & 5 & 10 & 20 & 30 \\
\hline WT & 322 & 322 & 322 & 323 & 325 & $4.0 \pm 0.3$ & $4.2 \pm 0.2$ & $4.4 \pm 0.6$ & $5 \pm 0.4$ & $5.5 \pm 0.3$ \\
\hline$\Delta \mathrm{N}$ & 319 & 322 & 324 & 325 & 325 & $7 \pm 0.4$ & $7.6 \pm 0.3$ & $8 \pm 0.3$ & $8.6 \pm 0.4$ & $8.8 \pm 0.6$ \\
\hline
\end{tabular}

${ }^{a}$ Parameters were derived from Trp fluorescence data shown in Fig. 3a

b The Stern-Volmer quenching constants were derived from the slopes of the linear regression lines from plots of $F_{0} / F=1+K_{\mathrm{Sv}}[\mathrm{Q}]$ shown in Fig. 4a. All values are the means \pm SD of three experiments

concentrations ( $\sim 40$ vol\%) did not change Trp fluorescence parameters significantly (not shown). These results indicate that KcsA tetramer is quite stable in a PE:PG bilayer and that the folding properties of KcsA are remarkably different in this lipid system compared to other artificial membrane systems studied previously (Raja et al. 2007).

Qualitatively, similar results were obtained for $\Delta \mathrm{N}-\mathrm{Kcs}$ A (Fig. 3b, Table 1) in a PE:PG (7:3) bilayer. Again, 5-10 vol\% TFE induced an increase in Trp fluorescence, and addition of 20-30 vol\% TFE resulted in fluorescence quenching (30-39\%). However, redshifts in emission maxima were found to be more pronounced in $\Delta \mathrm{N}-\mathrm{Kcs} \mathrm{A}$ as a function of TFE concentration. Taken into account changes in Trp fluorescence parameters in WT and $\Delta \mathrm{N}-\mathrm{Kcs} \mathrm{A}$ in both types of lipid bilayers the N-terminus seems to play a role in protecting KcsA tetramer from unfolding especially at lower TFE concentrations.

The increase and decrease in fluorescence as well as redshifts in emission maxima suggested that KcsA tetramer undergoes various conformational changes at low and high TFE concentrations. Thus, a more direct approach of investigating protein conformational changes by acrylamide quenching experiments was used. The main benefit of using acrylamide is that it is a hydrophilic quencher of Trp fluorescence and it has a quite low permeability to lipid membranes. In addition, no charge interaction takes place between the negatively charged lipid head groups (Breukink et al. 1998). The Stern-Volmer quenching plots of WT and $\Delta \mathrm{N}-\mathrm{KcsA}$ in a PE:PG lipid bilayer either in the absence and presence of TFE were found to be linear and are shown in Fig. 4a and b, respectively. The SternVolmer constants $\left(K_{\mathrm{SV}}\right)$ are compiled in Table 1 . In the absence of TFE, a significant increase in quenching constant for $\Delta \mathrm{N}-\mathrm{KcsA}$ compared to WT-KcsA was observed indicating that both tetramers are slightly different with regard to their membrane organization. For WT-KcsA (Fig. 4a), no significant change in acrylamide quenching was observed for 5-10 vol\% TFE; however, a slight increase in quenching was observed at 20-30 vol\% TFE indicating protein unfolding as also deduced by redshift in Trp fluorescence spectra (Fig. 3a). Interestingly, acrylamide quenching was found to be more pronounced in $\Delta \mathrm{N}-\mathrm{KcsA}$ (Fig. 4b) indicating that in the absence of $\mathrm{N}$-terminus the KcsA is slightly more sensitive to TFEinduced tetramer unfolding.

For comparison, the values derived from the effect of TFE on Trp fluorescence and acrylamide quenching experiments of WT-KcsA in a PC:PA (7:3) lipid bilayer from the previous studies (Raja et al. 2007) are compiled in Table 2. In the absence of TFE, Trp fluorescence emission wavelengths and Stern-Volmer quenching constants were found to be quite similar for WT-KcsA suggesting that the tetramer adopts a similar folding pattern both in PE:PG and PC:PA lipid bilayers (compare Table 1 and Table 2). However, after the addition of TFE, Trp fluorescence and quenching constants were found to be significantly different among both lipid bilayers indicating that in addition to
Fig. 4 Representative Stern-Volmer plots of Trp fluorescence quenching by acrylamide in WT-KcsA (a) and $\Delta \mathrm{N}-\mathrm{KcsA}(\mathbf{b})$ in PE:PG (7:3 mol\%) bilayers. Samples were investigated with or without certain concentrations of TFE (vol\%) as indicated. The slopes of the best fit linear regression lines for each data set ( $K_{\mathrm{SV}}$ values) are shown in Table 1
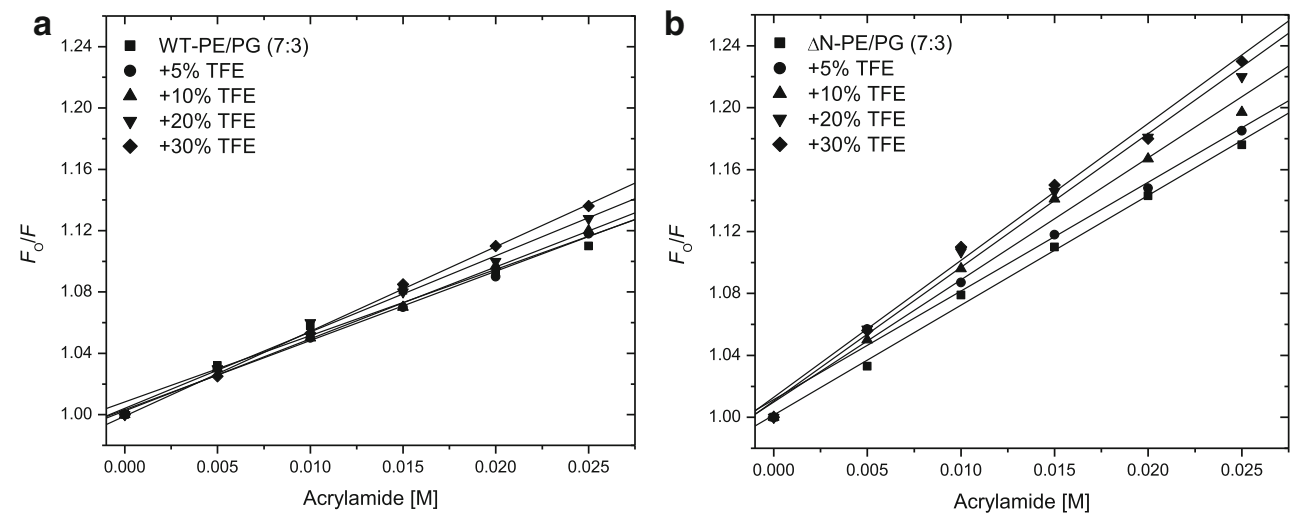
Table 2 Effects of TFE on folding properties of WT and $\Delta \mathrm{N}-\mathrm{KcsA}$ in a PC:PA (7:3 mol\%) lipid bilayer

\begin{tabular}{|c|c|c|c|c|c|c|c|c|c|c|}
\hline \multirow{2}{*}{$\frac{\text { Sample }}{\text { TFE (vol\%) }}$} & \multicolumn{5}{|c|}{ Wavelength $(\mathrm{nm})^{\mathrm{a}}$} & \multicolumn{5}{|c|}{$K_{\mathrm{SV}}\left(\mathrm{M}^{-1}\right)^{\mathrm{b}}$} \\
\hline & 0 & 5 & 10 & 20 & 30 & 0 & 5 & 10 & 20 & 30 \\
\hline $\mathrm{WT}^{\mathrm{c}}$ & 320 & 320 & 320 & 321.5 & 321.5 & $3 \pm 0.6$ & $2.6 \pm 0.8$ & $2.5 \pm 1.2$ & $2.7 \pm 0.8$ & $8.0 \pm 1.8$ \\
\hline$\Delta \mathrm{N}^{\mathrm{d}}$ & 320 & 320 & 320 & 320 & 320 & $2.2 \pm 0.7$ & $2.8 \pm 1$ & $4.5 \pm 0.8$ & $6.5 \pm 2.3$ & $7 \pm 1.6$ \\
\hline
\end{tabular}

H-bonding the strong ionic interaction between the negatively charged PA and positively charged residues on WTKcsA protects tetramer from unfolding.

For $\Delta \mathrm{N}-\mathrm{Kcs} \mathrm{A}$ in PC:PA (Table 2, data not shown), no change in Trp emission wavelength was observed either in the absence or presence of TFE. However, significantly reduced accessibility to acrylamide or decrease in SternVolmer quenching constant compared to $\Delta \mathrm{N}-\mathrm{Kcs} \mathrm{A}$ in PE:PG was calculated (compare Table 1 and Table 2). These results indicate that the $\mathrm{N}$-terminus significantly contributes to protect KcsA tetramer from unfolding as a result of its deep insertion into PE lipid headgroup. However, in PC:PA, the N-terminus seems to have a marginal role in protecting KcsA against TFE-induced unfolding because the positively charged region (Arg117, Arg121 or Arg122) close to the intracellular membrane surface at the C-terminus has a preferential interact with PA as also shown in the previous studies (Raja et al. 2007).

\section{Discussion}

Several interesting observations were made in the present study. First, the N-terminus significantly increased the tetrameric stability in a PE:PG (7:3 mol\%) lipid bilayer which mimics the natural membrane environment for KcsA assembly in bacterial membranes. The stabilizing effect of the N-terminus was similar to what has been shown in the previous studies (Raja 2010b, c) indicating that N-terminus indeed contributes to stabilize the tetrameric structure via electrostatic interaction regardless of the size of zwitterionic PE or PC headgroups.

An increase in Trp fluorescence at low concentrations of TFE was unique for PE:PG lipid bilayer system, because fluorescence quenching was observed for other membrane systems (Raja et al. 2007). Furthermore, TFE-induced Trp fluorescence quenching for PE:PG system was not as drastic as determined for pure PC or PC:PG lipid bilayers (Raja et al. 2007). In fact, Trp fluorescence and acrylamide quenching parameters were found to be quite close to
PC:PA lipid bilayer system (Raja et al. 2007). These observations suggest that the tetramer has a maximum stability in a PE:PG system. In other words, PE tends to protect KcsA tetramer from unfolding even at higher TFE concentrations.

It is important to mention here that PG, as a bilayer lipid, is required for the membrane stability of a PE:PG lipid bilayer because $\mathrm{PE}$ is a nonbilayer lipid and cannot form bilayer structures alone. It is known that in the PE:PG bilayer PE interacts more strongly with PG than with PE, thus controlling the bacterial membrane permeability and stability (Murzyn et al. 2005). Such information can also be supported by the fact that in additional experiments the PE:PA (7:3 mol\%) lipid combination resulted in a complete loss of KcsA protein incorporation efficiency indicating that no bilayer structures were formed for a PE:PA system that could support KcsA reconstitution (not shown). These observations also suggest that PE does not interact with PA because it may behave as a nonbilayer lipid as a result of its small headgroup size as PE.

The question arises whether TFE has an effect on bilayer integrity. One possibility is that the small headgroup of PE may increase the space available for partitioning of TFE at the lipid/water interface as shown by carboxyfluorescein (CF) leakage experiment in the earlier studies (van den Brink-van der Laan et al. 2004). Another reasonable explanation is that PE:PG bilayer system is more compact and resistant to TFE-induced membrane destabilization as a result of the preferential interaction of $\mathrm{PE}$ with $\mathrm{PG}$, as discussed above. In case of a PC:PA lipid bilayer, no significant difference has been observed in the $\mathrm{CF}$ leakage pattern compared to PC or PC:PG lipid bilayers (Raja et al. 2007).

What makes PE so extraordinary among other lipids? PE favors the deep incorporation of proteins because of its smaller headgroup size (Dowhan and Bogdanov 2009; Heller et al. 1997). PE molecules are known to restrict the dissipation of clustered phospholipids especially anionic phospholipids (Bazzi and Nelsestuen 1992). A similar phenomenon can be assumed by which PE may induce 
lateral clustering of anionic PG in a PE:PG lipid bilayer that allows PG cluster to surround KcsA via electrostatic interactions involving Arg64 and Arg89 at the extracellular surface, Arg11, Lys14, or Arg19 at the N-terminus, and Arg117, Arg121, or Arg122 close to the intracellular membrane surface. The functional and structural importance of these residues can be explained by the fact that deletion of these Arg residues as hot spots, particularly at the C-terminus, impairs KcsA assembly in a heterologous E. coli expression system which contains PE and PG lipids in the inner membrane (Molina et al. 2004). Thus, the extreme stability and tight packing of KcsA will be followed by binding of KcsA positively charged residues (Arg and/or Lys) to negatively charged PG and then the deep insertion of membrane-binding domains would take place by extensive hydrophobic interaction and $\mathrm{H}$-bonding between PE and KcsA, which is not possible for PC. The hypothetical models were derived to compare KcsA folding in PC, PE, or PA containing lipid bilayers (Fig. 5a, b). In PC, KcsA tetramer adopts a wobbly or loosely packed structure, whereas in bilayers containing either PE or PA the tetramer has a more compact structure as a result of the deep insertion of the lipid binding domains of KcsA.

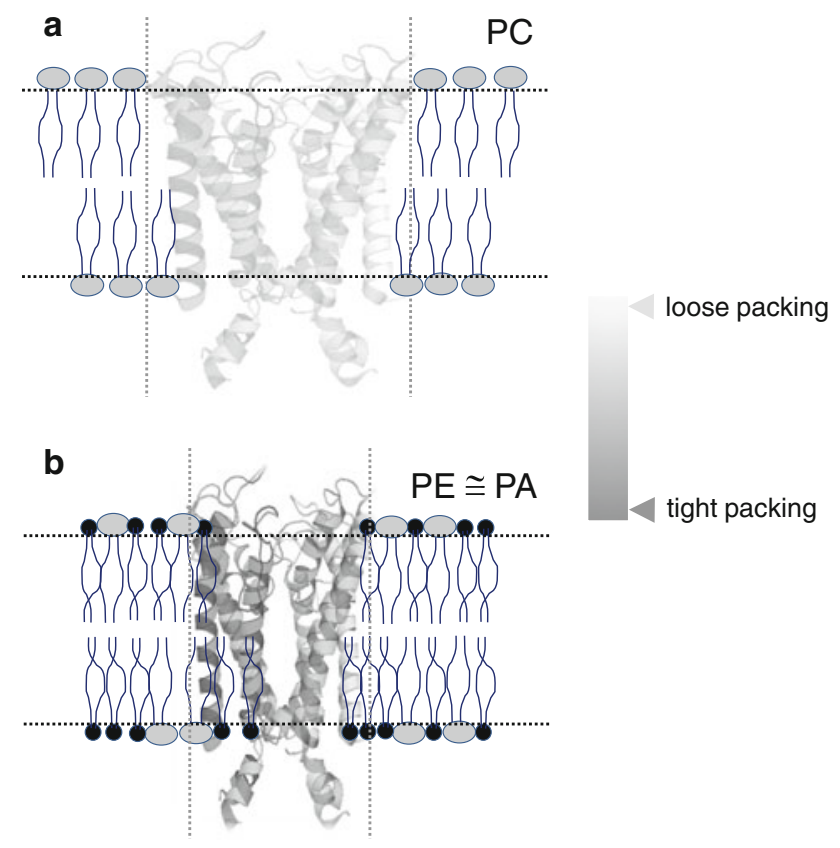

Fig. 5 A hypothetical scheme of KcsA tetramer folding in PC (a) or PE (b) containing lipid bilayers. In PC (large headgroup), the tetramer is loosely packed. However, in the presence of PE (small headgroup), the tetramer is squeezed and adopts a more compact structure similar to $\mathrm{PA}-\mathrm{KcsA}$ complex $(\mathrm{PE} \cong \mathrm{PA})$. The dotted lines represent a hypothetical scale to elaborate tetramer stretching or squeezing pattern in $\mathrm{PC}$ or $\mathrm{PE} \cong \mathrm{PA}$ lipid bilayers, respectively. The coloring of KcsA tetramer indicates the tetramer packing behavior. The $\mathrm{N}$ - and C-termini are not shown
In this context, the lipid headgroup properties of PE can be taken into further consideration. PE behaves as a cone-shaped lipid similar to the molecular shape of anionic PA (Kooijman et al. 2007). PE is known to facilitate membrane association and insertion of monomeric KcsA because of its small headgroup size (Ridder et al. 2001). Similarly, the small size of the PA headgroup as well as the negative charge allow stronger interaction and tight docking of the KcsA subunit into the membrane, thus increasing channel stability via H-bonding/electrostatic interactions (Raja et al. 2007). It is fascinating that both zwitterionic PE and anionic PA are completely different in their net charge. However, the folding and stabilizing effects of PE and PA on KcsA structure seem related, which might be due to the similar small headgroup size of both lipids.

A more in depth study varying the fatty acid composition of PE is still required to understand the effects of PE on integral membrane protein folding. However, the fatty acid compositions of PE and PC have been shown to have differing effects on other membrane proteins (Bogdanov et al. 1999, 2010), which are not yet completely understood.

Acknowledgments This work was supported by funds of the Chemical Sciences Division (CW) of the Netherlands Organization for Scientific Research (NWO). The useful comments of anonymous reviewers are gratefully acknowledged.

Open Access This article is distributed under the terms of the Creative Commons Attribution Noncommercial License which permits any noncommercial use, distribution, and reproduction in any medium, provided the original author(s) and source are credited.

\section{References}

Bazzi MD, Nelsestuen GL (1992) Interaction of annexin VI with membranes: highly restricted dissipation of clustered phospholipids in membranes containing phosphatidylethanolamine. Biochemistry-Us 31:10406-10413

Bogdanov M, Umeda M, Dowhan W (1999) Phospholipid-assisted refolding of an integral membrane protein. Minimum structural features for phosphatidylethanolamine to act as a molecular chaperone. J Biol Chem 274:12339-12345

Bogdanov M, Xie J, Heacock P, Dowhan W (2008) To flip or not to flip: lipid-protein charge interactions are a determinant of final membrane protein topology. J Cell Biol 182:925-935

Bogdanov M, Heacock P, Guan Z, Dowhan W (2010) Plasticity of lipid-protein interactions in the function and topogenesis of the membrane protein lactose permease from Escherichia coli. Proc Natl Acad Sci USA 107:15057-15062

Boggs JM (1987) Lipid intermolecular hydrogen bonding: influence on structural organization and membrane function. Biochim Biophys Acta 906:353-404

Breukink E, van Kraaij C, van Dalen A, Demel RA, Siezen RJ, de Kruijff B, Kuipers OP (1998) The orientation of nisin in membranes. Biochemistry-Us 37:8153-8162

Cortes DM, Cuello LG, Perozo E (2001) Molecular architecture of full-length KcsA-role of cytoplasmic domains in ion permeation and activation gating. J Gen Physiol 117:165-180 
Demmers JA, van Dalen A, de Kruijff B, Heck AJ, Killian JA (2003) Interaction of the $\mathrm{K}^{+}$channel KcsA with membrane phospholipids as studied by ESI mass spectrometry. FEBS Lett 541:28-32

der Laan E, Chupin V, Killian JA, de Kruijff B (2004) Stability of KcsA tetramer depends on membrane lateral pressure. Biochemistry-Us 43:4240-4250

Dill KA, Stigter D (1988) Lateral interactions among phosphatidylcholine and phosphatidylethanolamine head groups in phospholipid monolayers and bilayers. Biochemistry-Us 27:3446-3453

Dowhan W, Bogdanov M (2009) Lipid-dependent membrane protein topogenesis. Annu Rev Biochem 78:515-540

Heginbotham L, Kolmakova-Partensky L, Miller C (1998) Functional reconstitution of a prokaryotic $\mathrm{K}^{+}$channel. J Gen Physiol 111:741-749

Heller WT, He K, Ludtke SJ, Harroun TA, Huang HW (1997) Effect of changing the size of lipid headgroup on peptide insertion into membranes. Biophys J 73:239-244

Hope MJ, Bally MB, Webb G, Cullis PR (1985) Production of large unilamellar vesicles by a rapid extrusion procedure-characterization of size distribution, trapped volume and ability to maintain a membrane-potential. Biochim Biophys Acta 812:55-65

Kooijman EE, Tieleman DP, Testerink C, Munnik T, Rijkers DT, Burger KN, de Kruijff B (2007) An electrostatic/hydrogen bond switch as the basis for the specific interaction of phosphatidic acid with proteins. J Biol Chem 282:11356-11364

Molina ML, Encinar JA, Barrera FN, Fernandez-Ballester G, Riquelme G, Gonzalez-Ros JM (2004) Influence of C-terminal protein domains and protein-lipid interactions on tetramerization and stability of the potassium channel KcsA. Biochemistry-Us 43:14924-14931

Murzyn K, Rog T, Pasenkiewicz-Gierula M (2005) Phosphatidylethanolamine-phosphatidylglycerol bilayer as a model of the inner bacterial membrane. Biophys J 88:1091-1103
Raetz CR (1978) Enzymology, genetics, and regulation of membrane phospholipid synthesis in Escherichia coli. Microbiol Rev 42:614-659

Raja M (2010a) Monitoring the effects of strong cosolvent hexafluoroisopropanol in investigation of the tetrameric structure and stability of $\mathrm{K}(+)$-channel KcsA. Arch Biochem Biophys 498:1-6

Raja M (2010b) The role of extramembranous cytoplasmic termini in assembly and stability of the tetrameric $\mathrm{K}(+)$-channel KcsA. J Membr Biol 235:51-61

Raja M (2010c) The role of phosphatidic acid and cardiolipin in stability of the tetrameric assembly of potassium channel KcsA. J Membr Biol 234:235-240

Raja M, Spelbrink RE, de Kruijff B, Killian JA (2007) Phosphatidic acid plays a special role in stabilizing and folding of the tetrameric potassium channel KcsA. FEBS Lett 581:5715-5722

Ridder ANJA, Kuhn A, Killian JA, de Kruijff B (2001) Anionic lipids stimulate Sec-independent insertion of a membrane protein lacking charged amino acid side chains. EMBO Rep 2:403-408

Rietveld AG, Koorengevel MC, Dekruijff B (1995) Nonbilayer lipids are required for efficient protein-transport across the plasmamembrane of Escherichia coli. EMBO J 14:5506-5513

van Dalen A, Hegger S, Killian JA, de Kruijff B (2002) Influence of lipids on membrane assembly and stability of the potassium channel KcsA. FEBS Lett 525:33-38

van Klompenburg W, Paetzel M, de Jong JM, Dalbey RE, Demel RA, von Heijne G, de Kruijff B (1998) Phosphatidylethanolamine mediates insertion of the catalytic domain of leader peptidase in membranes. FEBS Lett 431:75-79 